\title{
ECOLOGICAL STUDY ON THREE MAJOR INSECT PESTS OF TOMATO PLANTATIONS IN FAYOUM GOVERNORATE.
}

\author{
Narmen .A. Youssef \\ Department of Plant Protection, Faculty of Agriculture, Fayoum \\ UNIVERSITY, FAYOUM, EGYPT
}

ABSTRACT

Tuta absoluta (Meyrick), Nesidiocoris tenuis (Reuter) and Bemisia tabaci (Genn.) activities on tomato plants at Fayoum Governorate were evident during (nili and summer 2011/2012 \& 2012/2013). The highest number of immature stages (larvae) of T.absuluta occurred in November \& December 2011 and 2012 (37 and 25 indiv./10 leaves) respectively during nili plantation, while the numbers of this insect were decreased during the summer plantation 2012 , the highest number recorded on June (20 indiv./10 leaves). There was highly significant correlation between the T.absuluta population and mean temp. during nili plantation 2011 . On the other hand, the highest populations of B. tabaci (97 and 70 indiv./10 leaves) was recorded in $2^{\text {nd }}$ and $4^{\text {th }}$ week of Oct. during (nili 2011/2012) and (81 and 64 nymphs/10 leaves) in $2^{\text {nd }}$ week of June and July (summer 2012/2013), respectively. For N. tenuis, the highest number (47 ; 39; 50 and 39 individuals/10 leaves) occurred during 1st and 2nd week of October during nili $2011 \& 2012$ and 2nd week of June, 1st week of July during summer 2012/2013, respectively. Also, six species of predators were recorded in association with tomato plants, Orius sp., Chrysopa vulgaris ,Syrphus corella, Coccinella undecimpunctata, Mantis religiosa and Paedrus alfierii.

Key words: Ecology Studies; Tuta absoluta ; Nesidiocoris tenuis ; Bemisia tabaci; Tomato.

\section{INTRODUCTION}

Tomato ,Lycopersicom esculentum $\mathrm{L}$. is the main host plant of different pests , namely : some lepidapterans Lepidoptera (leafminers), whiteflies, aphids and mealybug which cause considerable damage to both quantity and quality of the fruits (Faragalla, 2005 and Cheerapha 2005). According to Barrientos et al., 1998 and EPPO 2005 the tomato leafminer, Tuta absoluta (Meyrick), (Lepidoptera: Gelechiidae) is one of the most devastating pests for tomato crops.

due to the serious damage inflicted to tomato in invaded areas where infestation was initially detected (Germain et al., 2009). without control measures , the pest can cause up to $80-100 \%$ yield losses where leaves, flowers, stems and especially fruits were attacked (Lopez, 1991 and Andrew et al., 2013). the tomato plant bug, Nesidiocoris tenuis (Reuter) (Hemiptera: Miridae) 2 and the

Fayoum J. Agric. Res. \& Dev., Vol. 29, No.2, July, 2015 
tomato whitefly Bemisia tabaci (Genn.) (Hemiptera: Aleyrodidae) were also particularly important pests and were the major cause of the reduction in tomato production. (Cheerapha 2005). B. tabaci direct damage is by sucking and devitalizing of plants, besides the spreading of leaf curlvirus disease that leads to huge loss of crop. (Chaudhuri et al., 2001).

During the past few years, high infestations of these pests were noticed in different regions in Egypt including Fayoum, where tomato crop which is considered one of the economically important produce of this governorate.

Therefore, the aim of the present study is to evaluate the population dynamics of these three major pests in tomato plantations in Fayoum in relation to the major prevailing weather factors (mean temperatures and mean relative humidity) during nili and summer seasons of 2011- 2013.

\section{MATERIALS AND METHODS}

Experimentation was carried out in Al- Mandara region, Fayoum Governorate, Egypt to evaluate the population fluctuations of immature stages of T. absoluta, and B. tabaci and of the nymphs and adults of $N$. tenuis in tomato (variety Helal) during four successive nili and summer plantations 2011/2012 and 2012/2013.

For this purpose, a chosen area of about $1 / 4$ feddan was individed into four equal as a plots randomized complete block design. Tomato was cultivated as usual in two successive annual plantations (nili and summer seasons).

The data for the maximum and minimum temperatures and for relative humidity were obtained from the Meteorological Station of Fayoum Governorate. The usual agricultural practices were practiced excluding pesticide treatments.

\section{Estimation of population:}

Inspection of tomato leaf samples started two weeks after seedling plantation and continued weakely until the end of the season such samples were taken in the early morning, ten leaves per plot, picked randomly from different levels of the plants and separately transferred in special bags to the laboratory.

The number of pests and predators contained in each samples 3 was counted in the same day by the aid of a steremicroscope. The total number of individuals of $N$. tenuis (nymphs and adults), B. tabaci (nymphs stages) and predators present were also counted. T. absoluta was counted as larvae and empty mines on the leaves.

\section{Statistical analysis}

The relationship between the populations of the insects recorded and prevailing weather factors (Temp. \& R .H\%) were obtained by using the simple correlation, (Snedecor and Cochran, 1990).

Fayoum J. Agric. Res. \& Dev., Vol. 29, No.2, July, 2015 
ECOLOGICAL STUDY ON THREE MAJOR INSECT PESTS... ......... 3 RESULTS AND DISCUSSION

\section{A. Population dynamics of T. absoluta}

Nili plantations 2011 and 2012:

Few numbers appeared during the period from late October to mid November (2011) ranging between 2-7 indiv./10 leaves. The population increased in the last week of November till reached a peak (26 indiv. /101 eaves), and a 2 nd peak (37 indiv./10 leaves) on December 6 (2011). Positive highly significant correlation was found between the total population and mean temp. ( $\mathrm{r}=0.64 * *)$ and relative humidity $\left(\mathrm{r}=0.62^{* *}\right)$ as shown in table 1 and fig. 1 .Also in 2012 the 1 st but smaller peak (11 indiv. /10 leaves) on Oct.7. the 2nd peak occurred in late Nov. (25 indiv./10 leaves). There was no significant relation between the numbers of insects in this case and the means of temp. and R.H.\% ( $\mathrm{r}=-0.41$ and -0.24 ) as shown in table 1 and fig 1.

Table(1): Weekly numbers of Immature stage andmines of Tutauabsolute 11Tula absdutaleaves of tomato during Nili plantations 2011 \& 2012.

\begin{tabular}{|c|c|c|c|c|c|c|c|c|c|}
\hline \multicolumn{5}{|c|}{ Nili 2011} & \multicolumn{5}{|c|}{ Nili 2012} \\
\hline \multirow{2}{*}{$\begin{array}{c}\text { Sampling } \\
\text { date }\end{array}$} & \multirow{2}{*}{$\begin{array}{c}\text { Immature } \\
\text { (Larvae) }\end{array}$} & \multirow{2}{*}{\begin{tabular}{|c|} 
Mines \\
Empty \\
\end{tabular}} & \multicolumn{2}{|c|}{ Weather factors } & \multirow{2}{*}{\multicolumn{2}{|c|}{$\begin{array}{c}\text { Sampling Immature } \\
\text { date (Larvae) }\end{array}$}} & \multirow{2}{*}{$\begin{array}{l}\text { Mines } \\
\text { Empty }\end{array}$} & \multicolumn{2}{|c|}{ Weather factors } \\
\hline & & & $\begin{array}{c}\text { Mean } \\
\text { Temnc }\end{array}$ & $\begin{array}{l}\text { Mean } \\
\text { R.H\% }\end{array}$ & & & & \begin{tabular}{|c|} 
Mean \\
Temn.'c
\end{tabular} & $\begin{array}{l}\text { Mean } \\
\text { R.H\% }\end{array}$ \\
\hline $14 / 8 / 2011$ & 0 & 1 & 35 & 50 & 26/8/2012 & 0 & 0 & 31.3 & 50 \\
\hline 2118 & 0 & 0 & 31 & 48 & 319 & 0 & 0 & 30 & 49 \\
\hline $28 / 8$ & 1 & 3 & 30.7 & 47 & 10,9 & 0 & 0 & 29.6 & 50 \\
\hline $4 / 9$ & 0 & 1 & 29 & 50 & 1719 & 0 & 0 & 28.5 & 48 \\
\hline 1119 & 0 & 0 & 29.3 & 49 & 2319 & 0 & 1 & 29 & 50 \\
\hline $18 / 9$ & 0 & 0 & 29.4 & 46 & 30,9 & 0 & 0 & 29 & 52 \\
\hline $25 / 9$ & 0 & 0 & 28.9 & 49 & 7,10 & 11 & 0 & 29 & 52 \\
\hline 2110 & 0 & 0 & 26 & 51 & $14 / 10$ & 0 & 0 & 28.5 & 50 \\
\hline 9110 & 0 & 0 & 25.9 & 49 & 21110 & 0 & 0 & 29.8 & 48 \\
\hline $16 / 10$ & 0 & 0 & 24.7 & 49 & $28 / 10$ & 4 & 0 & 27.5 & 48 \\
\hline $25 / 10$ & 7 & 0 & 23 & 54 & 4111 & 0 & 0 & 27.5 & 47 \\
\hline 1111 & 4 & 2 & 22 & 53 & 11111 & 0 & 0 & 23 & 47 \\
\hline 8111 & 0 & 0 & 22.2 & 53 & $18 / 11$ & 1 & 0 & 20.5 & 45 \\
\hline $15 / 11$ & 2 & 8 & 19.5 & 55 & $25 / 11$ & 25 & 15 & 19.4 & 45 \\
\hline $22 / 11$ & 26 & 6 & 19.9 & 51 & $2 / 12$ & 4 & 9 & 19.4 & 45 \\
\hline $29 / 11$ & 9 & 3 & 17 & 56 & $9 / 12$ & 10 & 12 & 19.4 & 49 \\
\hline $6 / 12$ & 37 & 10 & 16.4 & 61 & $16 / 12$ & 5 & 18 & 17.7 & 49 \\
\hline 13112 & 23 & 15 & 15.4 & 62 & 23112 & 6 & 14 & 17.7 & 49 \\
\hline 21112 & 15 & 8 & 16.9 & 60 & 30,12 & 3 & 7 & 16.9 & 49 \\
\hline $27 / 12$ & 8 & 13 & 16.9 & 61 & $6 / 1 / 2013$ & 4 & 11 & 12 & 50 \\
\hline $4 / 1 / 2012$ & 3 & 6 & 15 & 61 & & & & & \\
\hline Total & 135 & 76 & & & Total & 73 & 87 & & \\
\hline Mean & 6.43 & 3.62 & & & Mean & 3.65 & 4.35 & & \\
\hline
\end{tabular}

Fayoum J. Agric. Res. \& Dev., Vol. 29, No.2, July, 2015 
Nili 2011

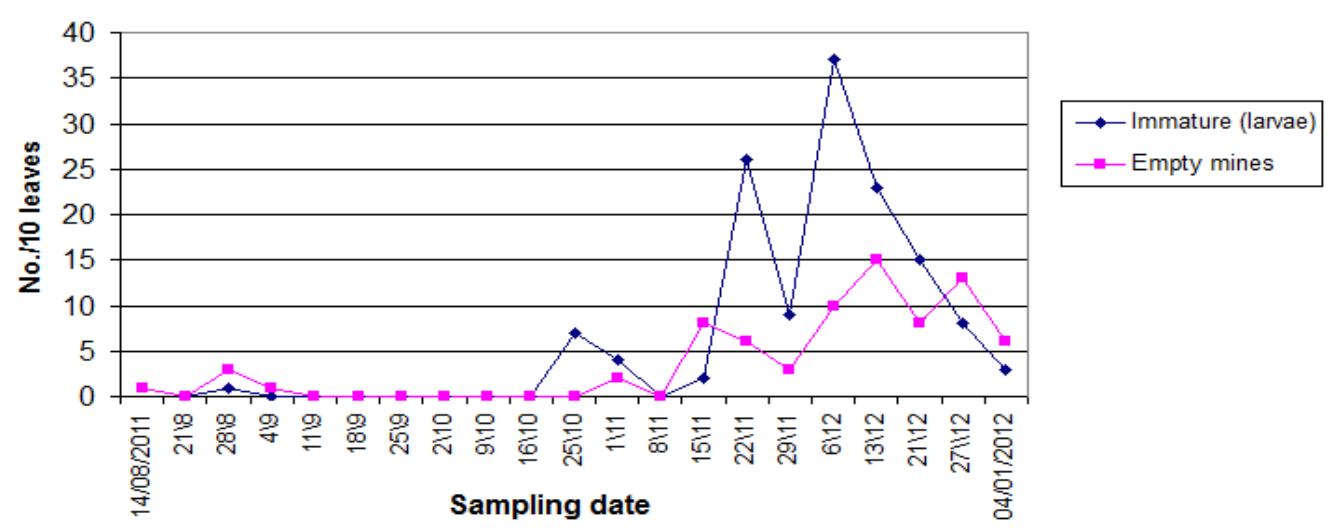

Nili 2012

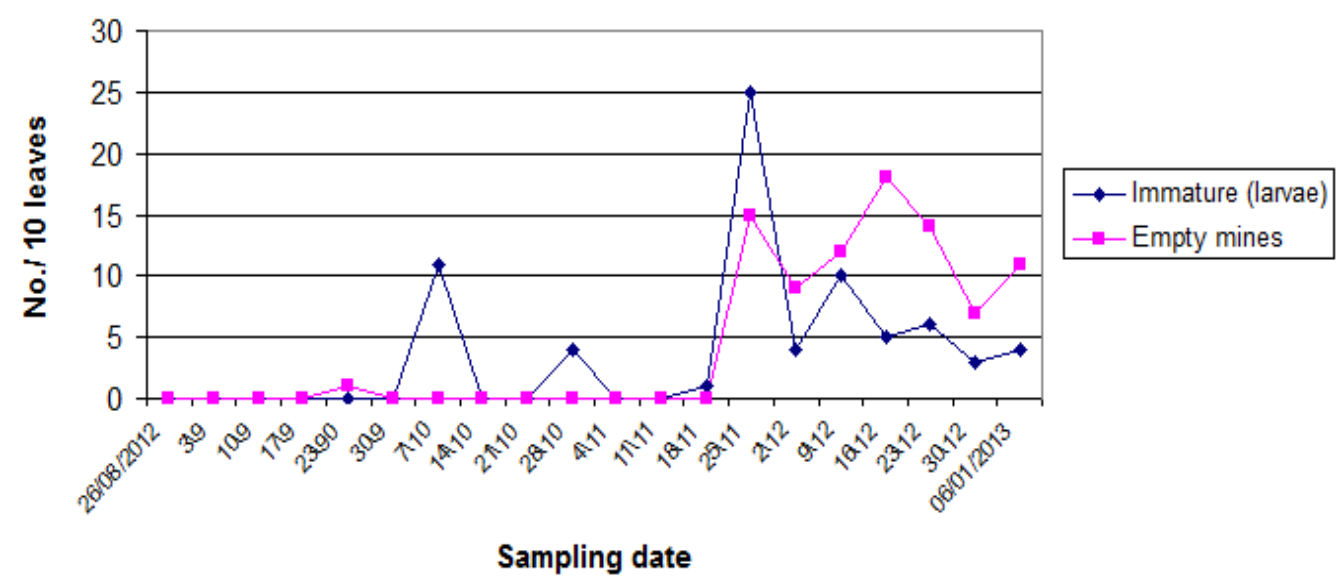

Fig. (1): Weekly numbers of Immature stage and mines of Tuta absoluta /10 leaves of tomato during Nili plantations 2011 \& 2012

\section{Summer plantations 2012 and 2013:}

This insect was mostly found during the period from Apr. 28 to July 9 of 2012. The 1 st peak occurred on Apr. 28 (10 indiv. /10 leaves), also the 2ndpeak was found on May 18 (14 indiv./10 plants) and the 3rd peak (20 indiv./10 leaves) occurred on June 10 .A positive insignificant correlation was found between the insect population and mean temp. and R.H. ( $\mathrm{r}=0.16$ and 0.054, respectively). During 2013, few numbers of this insect was found. The population fluctuated between 2 and 9 indiv./10 leaves. Also, insignificant correlation was found ( $\mathrm{r}=0.299$ and -0.24$)$ as shown in table 2 and fig 2 .

Fayoum J. Agric. Res. \& Dev., Vol. 29, No.2, July, 2015 
ECOLOGICAL STUDY ON THREE MAJOR INSECT PESTS... ........ 5

Table (2): Weekly numbers of Immature stage and mines of Tuta absoluta /10 leaves of tomato during Summer plantations $2012 \& 2013$.

\begin{tabular}{|c|c|c|c|c|c|c|c|c|c|}
\hline \multicolumn{5}{|c|}{ Summer 2012} & \multicolumn{5}{|c|}{ Summer 2013} \\
\hline \multirow{2}{*}{$\begin{array}{c}\text { Sampling } \\
\text { date }\end{array}$} & \multirow{2}{*}{$\begin{array}{c}\text { Immature } \\
\text { (Larvae) }\end{array}$} & \multirow{2}{*}{$\begin{array}{l}\text { Mines } \\
\text { Empty }\end{array}$} & \multicolumn{2}{|c|}{ Weather factors } & \multirow{2}{*}{$\begin{array}{l}\text { Sampling } \\
\text { date }\end{array}$} & \multirow{2}{*}{$\begin{array}{c}\text { Immature } \\
\text { (Larvae) }\end{array}$} & \multirow{2}{*}{$\begin{array}{l}\text { Mines } \\
\text { Empty }\end{array}$} & \multicolumn{2}{|c|}{ Weather factors } \\
\hline & & & $\begin{array}{c}\text { Mean } \\
\text { Temp.c }\end{array}$ & $\begin{array}{l}\text { Mean } \\
\text { R.H\% }\end{array}$ & & & & $\begin{array}{c}\text { Mean } \\
\text { Temp.c }\end{array}$ & $\begin{array}{l}\text { Mean } \\
\text { R.H\% }\end{array}$ \\
\hline $21 / 4 / 2012$ & 0 & 0 & 20.5 & 49 & $29 / 4 / 2013$ & 1 & 4 & 22.3 & 45 \\
\hline $28 / 4$ & 10 & 13 & 19 & 32 & $7 / 5$ & 0 & 2 & 25.4 & 45 \\
\hline $4 / 5$ & 5 & 7 & 22.9 & 48 & $16 / 5$ & 0 & 0 & 29.3 & 43 \\
\hline $11 / 5$ & 4 & 5 & 23.6 & 43 & $22 / 5$ & 3 & 10 & 28 & 43 \\
\hline $18 / 5$ & 14 & 8 & 27.7 & 52 & $29 / 5$ & 3 & 7 & 30 & 41 \\
\hline $26 / 5$ & 7 & 1 & 29.7 & 42 & $5 / 6$ & 2 & 1 & 31.8 & 41 \\
\hline $3 / 6$ & 6 & 2 & 27 & 44 & $12 / 6$ & 0 & 1 & 32.8 & 42 \\
\hline $10 / 6$ & 20 & 13 & 29 & 47 & $19 / 6$ & 7 & 13 & 30.8 & 43 \\
\hline $17 / 6$ & 14 & 27 & 30.5 & 43 & $26 / 6$ & 5 & 5 & 30 & 45 \\
\hline $24 / 6$ & 7 & 10 & 31 & 42 & $3 / 7$ & 9 & 7 & 32.3 & 44 \\
\hline $1 / 7$ & 5 & 8 & 31.4 & 46 & $9 / 7$ & 0 & 0 & 29.8 & 45 \\
\hline $9 / 7$ & 4 & 7 & 31 & 49 & $16 / 7$ & 0 & 0 & 31 & 47 \\
\hline $16 / 7$ & 0 & 1 & 30.7 & 40 & $23 / 7$ & 0 & 2 & 30 & 48 \\
\hline Total & 96 & 102 & & & Total & 30 & 52 & & \\
\hline Mean & 7.38 & 7.85 & & & Mean & 2.3 & 4 & & \\
\hline
\end{tabular}

Fayoum J. Agric. Res. \& Dev., Vol. 29, No.2, July, 2015 


\section{Summer 2012}

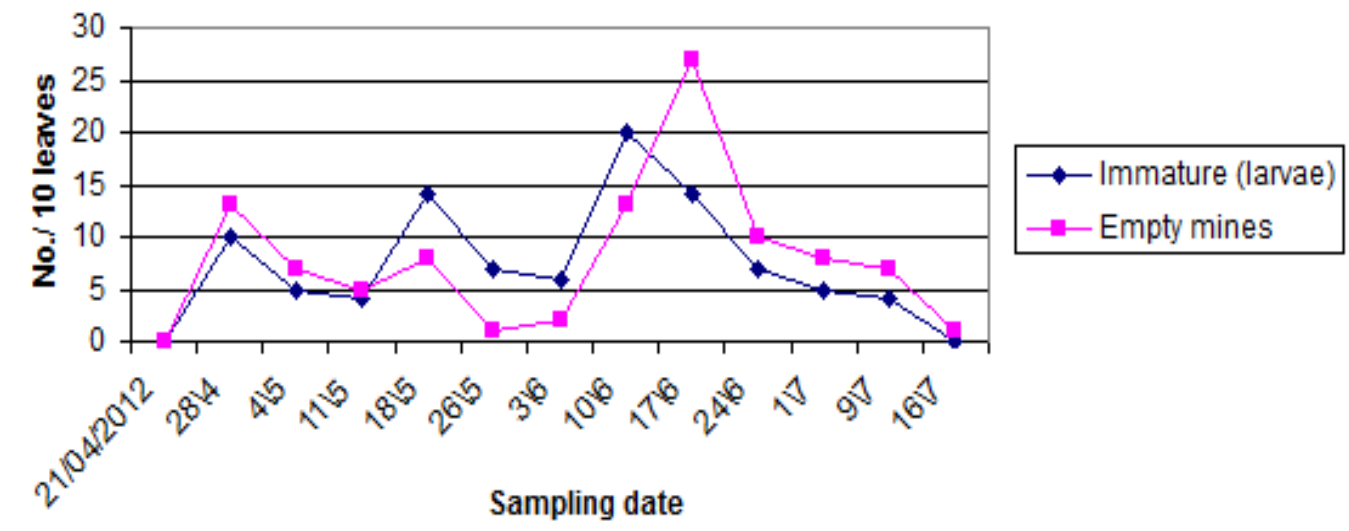

Summer 2013

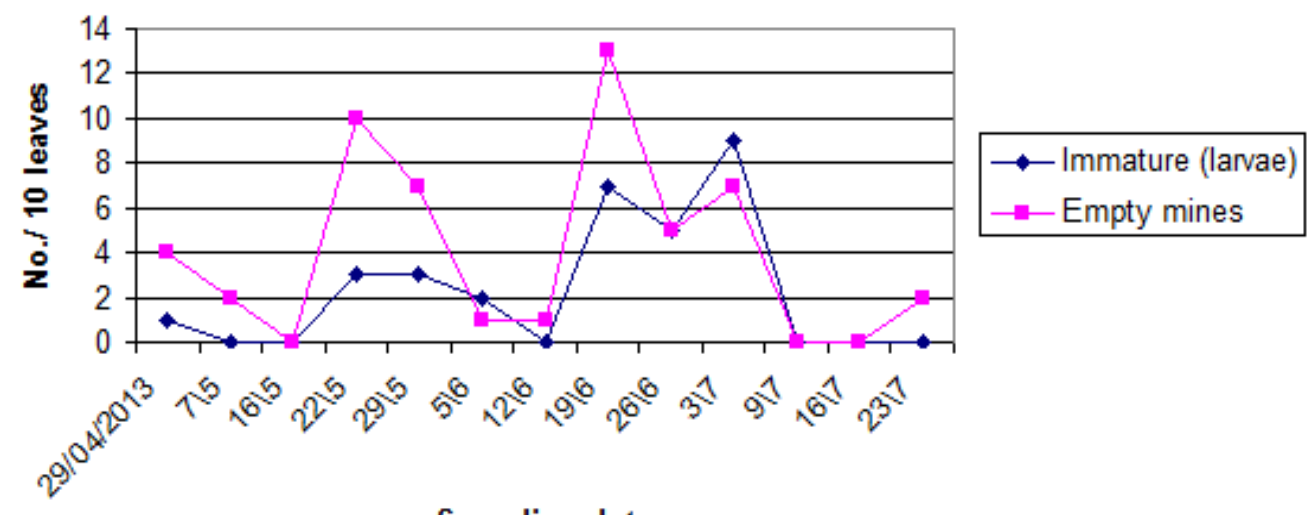

Sampling date

Fig. (2): Weekly numbers of Immature stage and mines of Tuta absoluta /10 leaves of tomato during Summer plantations 2012\& 2013.

Similar result was reported by Lazgeen $\boldsymbol{e t}$ al., 2013 in Iraq. where the number of mines started low in May and June and increased during August and September. The maximum number of leafminer strating from late July increasing of larvae numbers on leaves caused high infestation (72-100 \%) in September.

Also Nannini et al, (2011) in Italy found that the highest levels of tomato borer infestation occure in spring (30- 100 larvae/plant).

Fayoum J. Agric. Res. \& Dev., Vol. 29, No.2, July, 2015 
ECOLOGICAL STUDY ON THREE MAJOR INSECT PESTS... ......... 7

B. Population dynamics of $B$. tabaci

Nili population 2011 and 2012:

Infestation was observed in samples taken during the period from Sept. 4 to Nov. 22, 2011. The 1st peak occurred on Sept. 18 (81 indiv./10 leaves) and the 2nd peak on Oct 9 (97 indiv./10 leaves). Insignificant correlation was found between this population density and mean temp. $(\mathrm{r}=0.39)$ with a negative significant effect of relative humidity $(\mathrm{r}=-0.51)$.

In 2012, the population began with 5 indiv./10 leaves on Sept. 3 , then fluctuated reaching the 1st peak on Oct. 14 (66 indiv./10 leaves), and the 2 nd peak on Oct. 28 (70 indiv./10 leaves) then later decreased to 3 indiv./10 leaves on Dec. 2nd. A positive highly significant correlation was found between population and mean temp. $(\mathrm{r}=0.57 * *)$. Insignificant with relative humidity effect $(\mathrm{r}=0.31)$. as shown in table 3 and fig. 3 .

Table (3): Weekly numbers of Bemisia tabaci \&Nesidocoris tenuis /10 leaves of tomato during Nili 2011 \& 2012

\begin{tabular}{|c|c|c|c|c|c|c|c|c|c|}
\hline \multicolumn{5}{|c|}{ Nili 2011} & \multicolumn{5}{|c|}{ Nili 2012} \\
\hline \multirow{2}{*}{$\begin{array}{c}\text { Sampling } \\
\text { date }\end{array}$} & \multirow{2}{*}{$\begin{array}{c}\text { B. } \\
\text { tabaci } \\
\text { (nymph) }\end{array}$} & \multirow{2}{*}{$\begin{array}{c}N . \\
\text { tenuis } \\
(\mathrm{N}+\mathrm{A})\end{array}$} & \multicolumn{2}{|c|}{ Weather factors } & \multirow{2}{*}{$\begin{array}{c}\text { Sampling } \\
\text { date }\end{array}$} & \multirow{2}{*}{$\begin{array}{c}B . \\
\text { tabaci } \\
\text { (nymph) }\end{array}$} & \multirow{2}{*}{$\begin{array}{c}N . \\
\text { tenuis } \\
(\mathrm{N}+\mathrm{A})\end{array}$} & \multicolumn{2}{|c|}{ Weather factors } \\
\hline & & & $\begin{array}{c}\text { Mean } \\
\text { Temp.c }\end{array}$ & $\begin{array}{l}\text { Mean } \\
\text { R.H\% }\end{array}$ & & & & $\begin{array}{c}\text { Mean } \\
\text { Temp.c }\end{array}$ & $\begin{array}{l}\text { Mean } \\
\text { R.H\% }\end{array}$ \\
\hline $14 / 8 / 2011$ & 0 & 5 & 31 & 50 & $26 / 8 / 2012$ & 0 & 3 & 31.3 & 50 \\
\hline $21 / 8$ & 0 & 12 & 31 & 48 & $3 / 9$ & 5 & 7 & 30 & 49 \\
\hline $28 / 8$ & 0 & 11 & 30.7 & 47 & $10 / 9$ & 17 & 13 & 29.6 & 50 \\
\hline $4 / 9$ & 3 & 24 & 29 & 50 & $17 / 9$ & 19 & 16 & 28.5 & 48 \\
\hline $11 / 9$ & 26 & 22 & 29.3 & 49 & $23 / 9$ & 9 & 15 & 29 & 50 \\
\hline $18 / 9$ & 81 & 33 & 29.4 & 46 & $30 / 9$ & 55 & 15 & 29 & 52 \\
\hline $25 / 9$ & 78 & 34 & 28.9 & 49 & $7 / 10$ & 60 & 26 & 29 & 52 \\
\hline $2 / 10$ & 83 & 47 & 26 & 51 & $14 / 10$ & 66 & 39 & 28.5 & 50 \\
\hline $9 / 10$ & 97 & 13 & 25.9 & 49 & $21 / 10$ & 50 & 5 & 29.8 & 48 \\
\hline $16 / 10$ & 79 & 7 & 24.7 & 49 & $28 / 10$ & 70 & 5 & 27.5 & 48 \\
\hline $25 / 10$ & 37 & 5 & 23 & 54 & $4 / 11$ & 40 & 6 & 27.5 & 47 \\
\hline $1 / 11$ & 29 & 0 & 22 & 53 & $11 / 11$ & 15 & 3 & 23 & 47 \\
\hline $8 / 11$ & 10 & 0 & 22.2 & 53 & $18 / 11$ & 7 & 1 & 20.5 & 45 \\
\hline $15 / 11$ & 2 & 0 & 19.5 & 55 & $25 / 11$ & 3 & 7 & 19.4 & 45 \\
\hline $22 / 11$ & 2 & 2 & 19.9 & 51 & $2 / 12$ & 3 & 2 & 19.4 & 45 \\
\hline $29 / 11$ & 0 & 0 & 17 & 56 & $9 / 12$ & 0 & 5 & 19.4 & 49 \\
\hline $6 / 12$ & 1 & 2 & 16.4 & 61 & $16 / 12$ & 0 & 3 & 17.7 & 49 \\
\hline $13 / 12$ & 0 & 0 & 15.4 & 62 & $23 / 12$ & 0 & 1 & 17.7 & 49 \\
\hline $21 / 12$ & 0 & 0 & 16.9 & 60 & $30 / 12$ & 0 & 0 & 16.9 & 49 \\
\hline $27 / 12$ & 0 & 0 & 16.9 & 61 & $6 / 1 / 2013$ & 0 & 0 & 12 & 50 \\
\hline $4 / 1 / 2012$ & 0 & 0 & 15 & 61 & & & & & \\
\hline Total & 528 & 217 & & & Total & 419 & 172 & & \\
\hline Mean & 25.14 & 10.33 & & & Mean & 20.95 & 8.6 & & \\
\hline
\end{tabular}

$\mathrm{N}=$ nymph $\mathrm{A}=$ Adult

Fayoum J. Agric. Res. \& Dev., Vol. 29, No.2, July, 2015 
Nili 2011

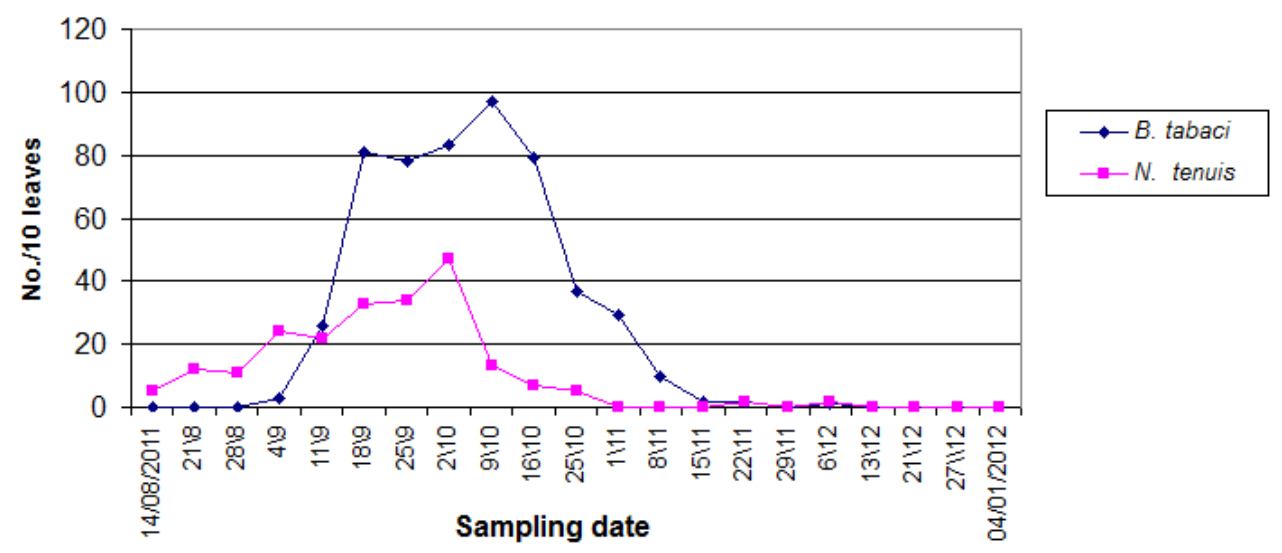

Nili 2012

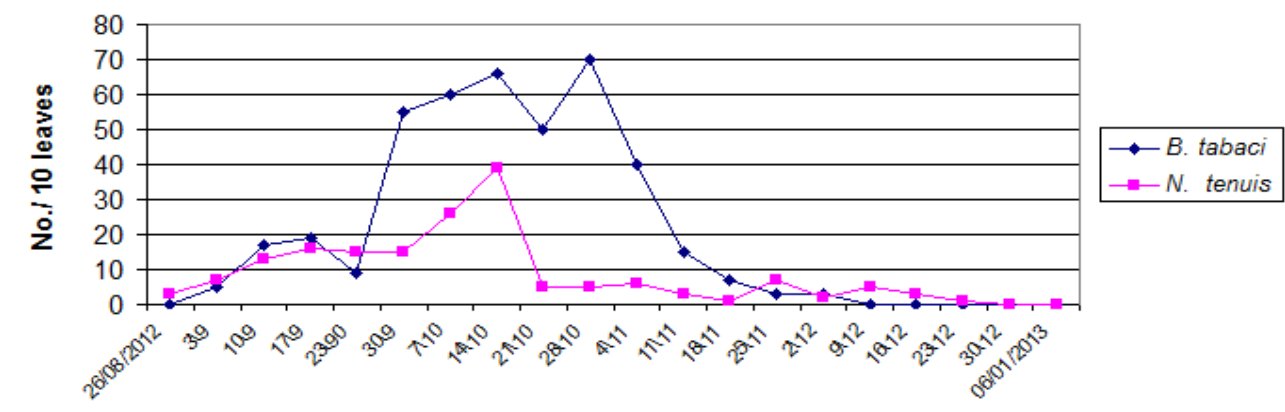

Sampling date

Fig. (3): Weekly numbers of Bemisia tabaci \& Nesidocoris tenuis /10 leaves of tomato during Nili 2011 \& 2012

\section{Summer population 2012 and 2013:}

Throughout the period from Apr. 21 to June 24 (2012), the population fluctuated reaching a single peak in June 10 (81 indiv./10 leaves). The correlation between population and mean temp. $\mathrm{r}=0.078$, and R.H.\% was insignificant with $\mathrm{r}=0.11$.

Infestation in 2013 was spread, allover the season. The highest density occurred during June and July with two peaks on June 12 and July 9 (44 and 64 indiv./10 leaves) respectively. Positive highly significant correlation was found between . population and mean temp. $(\mathrm{r}=0.71 * *)$ mean while, for relative humidity a negative insignificant correlation was found with $r=-0.032$ (Table 4 fig 4).

Fayoum J. Agric. Res. \& Dev., Vol. 29, No.2, July, 2015 
ECOLOGICAL STUDY ON THREE MAJOR INSECT PESTS... ........9

Table (4): Weekly numbers of Bemisia tabaci \& Nesidocoris tenuis /10 leaves of tomato during Summer 2012 \& 2013

\begin{tabular}{|c|c|c|c|c|c|c|c|c|c|}
\hline \multirow{3}{*}{$\begin{array}{c}\text { Sampling } \\
\text { date }\end{array}$} & \multicolumn{4}{|c|}{ Summer 2012} & \multirow{3}{*}{$\begin{array}{l}\text { Sampling } \\
\text { date }\end{array}$} & \multicolumn{4}{|c|}{ Summer 2013} \\
\hline & \multirow[t]{2}{*}{$\begin{array}{l}\text { B. tabaci } \\
\text { (nymph) }\end{array}$} & \multirow{2}{*}{$\begin{array}{c}\mathrm{N} . \\
\text { tenuis } \\
(\mathrm{N}+\mathrm{A})\end{array}$} & \multicolumn{2}{|c|}{$\begin{array}{c}\text { Weather } \\
\text { factors }\end{array}$} & & \multirow[t]{2}{*}{$\begin{array}{l}\text { B. tabaci } \\
\text { (nymph) }\end{array}$} & \multirow{2}{*}{$\begin{array}{c}\mathrm{N} . \\
\text { tenuis } \\
(\mathrm{N}+\mathrm{A})\end{array}$} & \multicolumn{2}{|c|}{$\begin{array}{c}\text { Weather } \\
\text { factors }\end{array}$} \\
\hline & & & $\begin{array}{c}\text { Mean } \\
\text { Temp.c }\end{array}$ & $\begin{array}{l}\text { Mean } \\
\text { R.H\% }\end{array}$ & & & & $\begin{array}{c}\text { Mean } \\
\text { Temp.c }\end{array}$ & $\begin{array}{l}\text { Mean } \\
\text { R.H\% }\end{array}$ \\
\hline $21 / 4 / 2012$ & 7 & 4 & 20.5 & 49 & $29 / 4 / 2013$ & 0 & 0 & 22.3 & 45 \\
\hline $28 / 4$ & 13 & 19 & 19 & 32 & $7 / 5$ & 0 & 15 & 25.4 & 45 \\
\hline $4 / 5$ & 10 & 29 & 22.9 & 48 & $16 / 5$ & 12 & 23 & 29.3 & 43 \\
\hline $11 / 5$ & 29 & 33 & 23.6 & 43 & $22 / 5$ & 29 & 28 & 28 & 43 \\
\hline $18 / 5$ & 50 & 37 & 27.7 & 52 & $29 / 5$ & 21 & 27 & 30 & 41 \\
\hline $26 / 5$ & 70 & 14 & 29.7 & 42 & $5 / 6$ & 30 & 4 & 31.8 & 41 \\
\hline $3 / 6$ & 77 & 0 & 27 & 44 & $12 / 6$ & 44 & 7 & 32.8 & 42 \\
\hline $10 / 6$ & 81 & 4 & 29 & 47 & $19 / 6$ & 40 & 13 & 30.8 & 43 \\
\hline $17 / 6$ & 23 & 50 & 30.5 & 43 & $26 / 6$ & 49 & 5 & 30 & 45 \\
\hline $24 / 6$ & 8 & 20 & 31 & 42 & $3 / 7$ & 62 & 39 & 32.3 & 44 \\
\hline $1 / 7$ & 0 & 27 & 31.4 & 46 & $9 / 7$ & 64 & 24 & 29.8 & 45 \\
\hline $9 / 7$ & 0 & 28 & 31 & 49 & $16 / 7$ & 40 & 4 & 31 & 47 \\
\hline $16 / 7$ & 0 & 13 & 30.7 & 40 & $23 / 7$ & 18 & 1 & 30 & 48 \\
\hline Total & 368 & 278 & & & Total & 409 & 190 & & \\
\hline Mean & 28.31 & 21.38 & & & Mean & 31.46 & 14.62 & & \\
\hline
\end{tabular}

Fayoum J. Agric. Res. \& Dev., Vol. 29, No.2, July, 2015 
Summer 2012

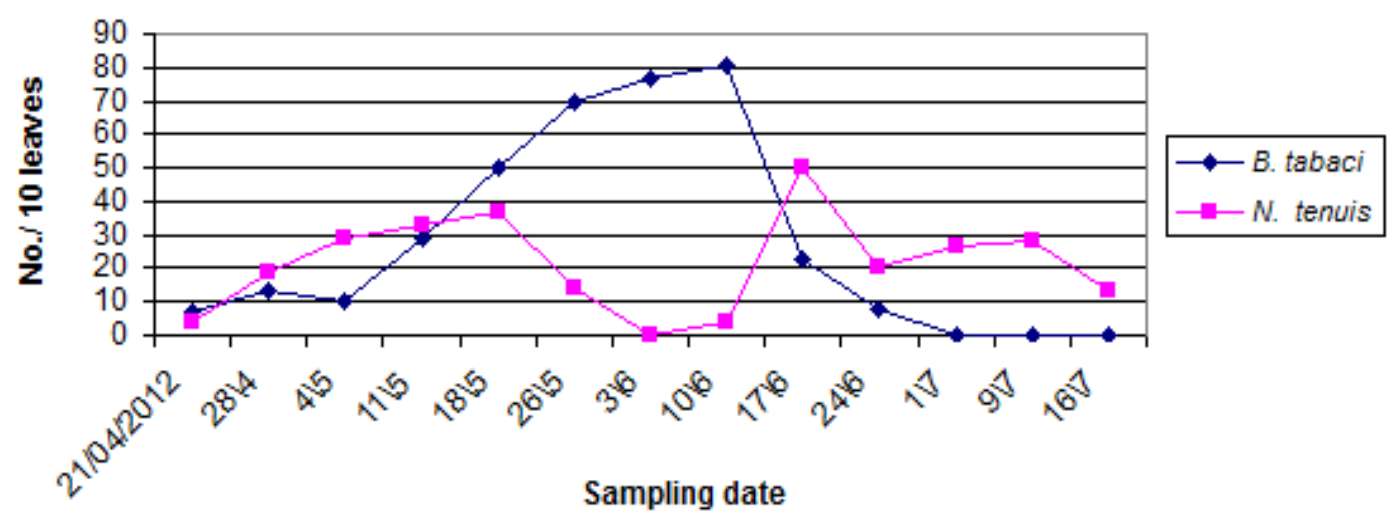

Summer 2013

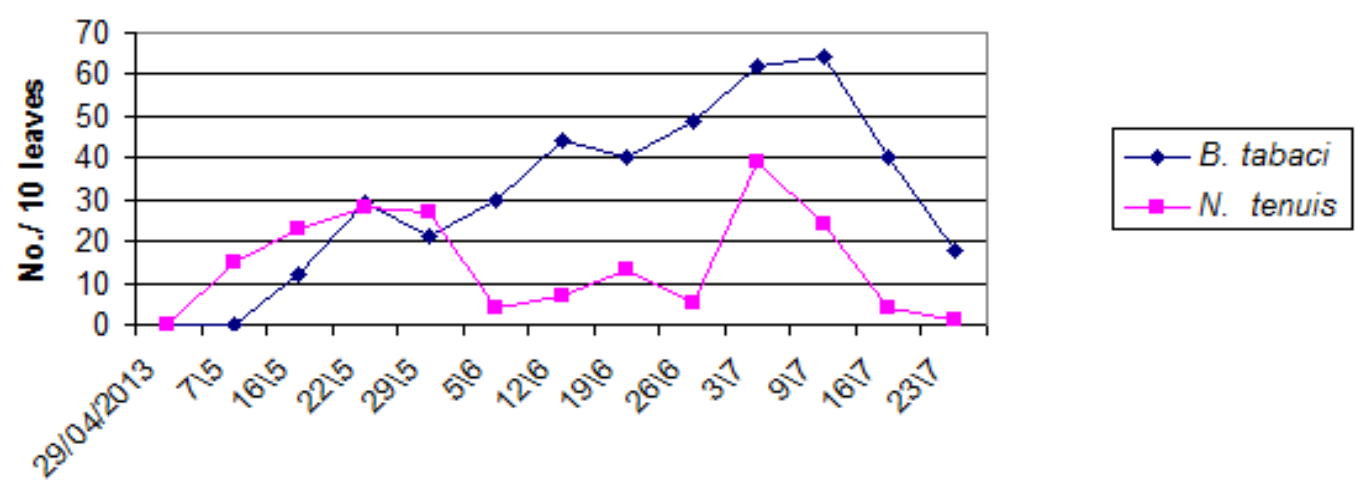

Sampling date

Fig. (4): Weekly numbers of Bemisia tabaci \& Nesidocoris tenuis /10 leaves of tomato during Summer 2012 \& 2013

In this respect contradicting results were reported by several investigated in Egypt where largest counts were observed in September;August to October;September to November and September by (Shanab and AwadAllah, 1982);(Shaheen, 1983; Shalaby et al., 1990) (Abd- Ellah, 1994) and (Tantaway, 1995), respectively.

Fayoum J. Agric. Res. \& Dev., Vol. 29, No.2, July, 2015 
ECOLOGICAL STUDY ON THREE MAJOR INSECT PESTS... ......... 11 C-Population dynamics of $N$. tenuis

Nili population 2011 and 2012 :

This tomato bug was abundant during the period from Aug. 14 to Oct. 25 (2011). With two peaks ; the 1st occurred in Sept. 4 with 24 indiv. $/ 10$ leaves , and the 2nd peak occurred on Oct. 2 with 47 indiv./ 10 leaves . Highly significant correlation was found between the population density of this insect species and mean temp. and relative humidity $(\mathrm{r}=0.64 * *$ and $-0.59 * *$ respectively).

The population fluctuated but in few numbers, with a small peak (16 indiv./10 leaves) on Sept. 17, and a higher peak on Oct. 14 (39 indiv./10 leaves).Highly significant correlation was found between population and mean temp. $\left(\mathrm{r}=0.58^{* *}\right)$ whereas the relationship with relative humidity was positively significant with $\mathrm{r}=0.5^{*}$ (Table 3 fig 3 ).

\section{Summer population 2012 and 2013 :}

During summer plantation 2012, the insect was spread allover the summer season. The highest density occurred in mid May and mid June with two peaks on May 15 and June 17 (37 and 50 indiv./10 leaves, respectively). Positive insignificant correlation was found between population and both mean temp. and relative humidity ( $\mathrm{r}=0.17$ and 0.11 , respectively).

In summer plantation 2013, infestation occurred from the 1st week of May to late July, with two peaks, on May 22 (28 indiv. / 10 leaves) and on July (39 indiv./10 leaves). Positive insignificant correlation was found between populationboth mean temp. and relative humidity $(\mathrm{r}=0.18$ and -0.35 , respectively) as shown in table 4 fig 4.

Similar corelations were reported by AL-Azawi and AL-Azawi (1988) in Iraq and Abd - Ellah (1994) in Egypt, this tomato bug attacks tomato plants nearly all over the year, and that population 14 fluctuation was high during summer season (June - September) then the rates of infestation drop sharply during December and January.

D- The relationship between insect population and predators: Nili plantations 2011 and 2012:

The population of predaceous insects was relatively at low level during nili plantations $2011 \& 2012$, therefore predation was not effective against the insect pests. as shown in Table 5 Fig 5.

Fayoum J. Agric. Res. \& Dev., Vol. 29, No.2, July, 2015 
Narmen .A. Youssef

Table (5): Weekly numbers of tomato pests and associated natural enemies during Nili plantations 2011 \& 2012

\begin{tabular}{|c|c|c|c|c|c|c|c|c|c|}
\hline \multicolumn{5}{|c|}{ Nili 2011} & \multicolumn{5}{|c|}{ Nili 2012} \\
\hline $\begin{array}{c}\text { Sampling } \\
\text { date }\end{array}$ & $\begin{array}{c}T . \\
\text { absoluta }\end{array}$ & $\begin{array}{c}\text { B. } \\
\text { tabaci }\end{array}$ & $\begin{array}{c}N . \\
\text { tenuis }\end{array}$ & $\begin{array}{l}\text { Natural } \\
\text { enemies }\end{array}$ & $\begin{array}{c}\text { Sampling } \\
\text { date }\end{array}$ & $\begin{array}{c}T . \\
\text { absoluta }\end{array}$ & $\begin{array}{c}B . \\
\text { tabaci }\end{array}$ & $\begin{array}{c}N . \\
\text { tenuis }\end{array}$ & $\begin{array}{l}\text { Natural } \\
\text { enemies }\end{array}$ \\
\hline $14 / 8 / 2011$ & 0 & 0 & 5 & 0 & $26 / 8 / 2012$ & 0 & 0 & 3 & 0 \\
\hline $21 / 8$ & 0 & 0 & 12 & 0 & $3 / 9$ & 0 & 5 & 7 & 0 \\
\hline $28 / 8$ & 1 & 0 & 11 & 0 & $10 / 9$ & 0 & 17 & 13 & 0 \\
\hline $4 / 9$ & 0 & 3 & 24 & 0 & $17 / 9$ & 0 & 19 & 16 & 0 \\
\hline $11 / 9$ & 0 & 26 & 22 & 0 & $23 / 9$ & 0 & 9 & 15 & 0 \\
\hline $18 / 9$ & 0 & 81 & 33 & 1 & $30 / 9$ & 0 & 55 & 15 & 1 \\
\hline $25 / 9$ & 0 & 78 & 34 & 1 & $7 / 10$ & 11 & 60 & 26 & 2 \\
\hline $2 / 10$ & 0 & 83 & 47 & 1 & $14 / 10$ & 0 & 66 & 39 & 0 \\
\hline $9 / 10$ & 0 & 97 & 13 & 0 & $21 / 10$ & 0 & 50 & 5 & 0 \\
\hline $16 / 10$ & 0 & 79 & 7 & 0 & $28 / 10$ & 4 & 70 & 5 & 1 \\
\hline $25 / 10$ & 7 & 37 & 5 & 1 & $4 / 11$ & 0 & 40 & 6 & 0 \\
\hline $1 / 11$ & 4 & 29 & 0 & 0 & $11 / 11$ & 0 & 15 & 3 & 0 \\
\hline $8 / 11$ & 0 & 10 & 0 & 0 & $18 / 11$ & 1 & 7 & 1 & 1 \\
\hline $15 / 11$ & 2 & 2 & 0 & 0 & $25 / 11$ & 25 & 3 & 7 & 4 \\
\hline $22 / 11$ & 26 & 2 & 2 & 0 & $2 / 12$ & 4 & 3 & 2 & 2 \\
\hline $29 / 11$ & 9 & 0 & 0 & 1 & $9 / 12$ & 10 & 0 & 5 & 2 \\
\hline $6 / 12$ & 37 & 1 & 2 & 0 & $16 / 12$ & 5 & 0 & 3 & 0 \\
\hline $13 / 12$ & 23 & 0 & 0 & 0 & $23 / 12$ & 6 & 0 & 1 & 0 \\
\hline $21 / 12$ & 15 & 0 & 0 & 0 & $30 / 12$ & 3 & 0 & 0 & 0 \\
\hline $27 / 12$ & 8 & 0 & 0 & 2 & $6 / 1 / 2013$ & 4 & 0 & 0 & 0 \\
\hline $4 / 1 / 2012$ & 3 & 0 & 0 & 0 & & & & & \\
\hline Total & 135 & 528 & 217 & 7 & Total & 73 & 419 & 172 & 13 \\
\hline Mean & 6.43 & 25.14 & 10.33 & 0.33 & Mean & 3.65 & 20.95 & 8.6 & 0.65 \\
\hline
\end{tabular}

Fayoum J. Agric. Res. \& Dev., Vol. 29, No.2, July, 2015 


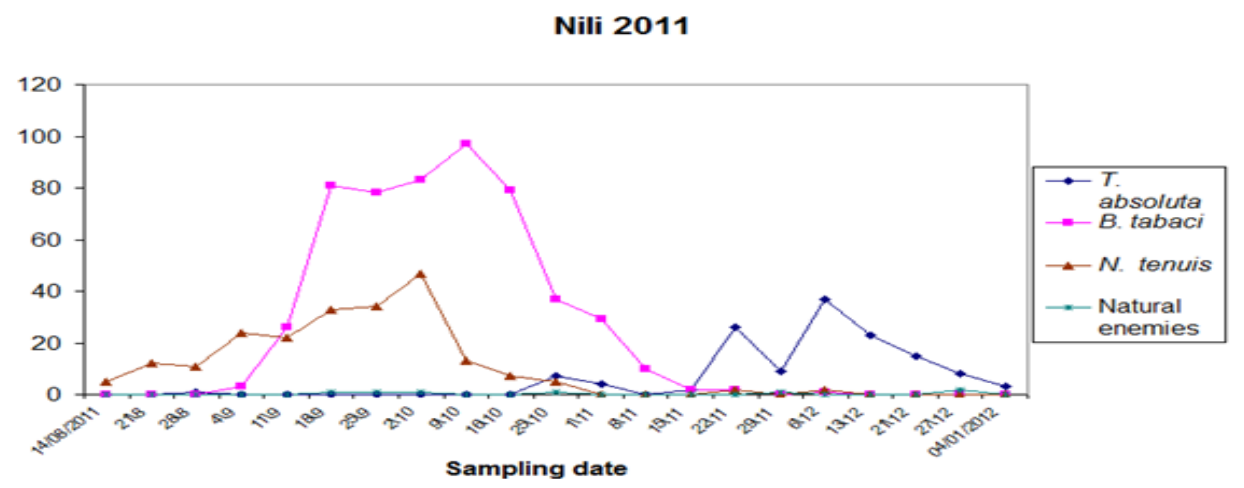

Nili 2012

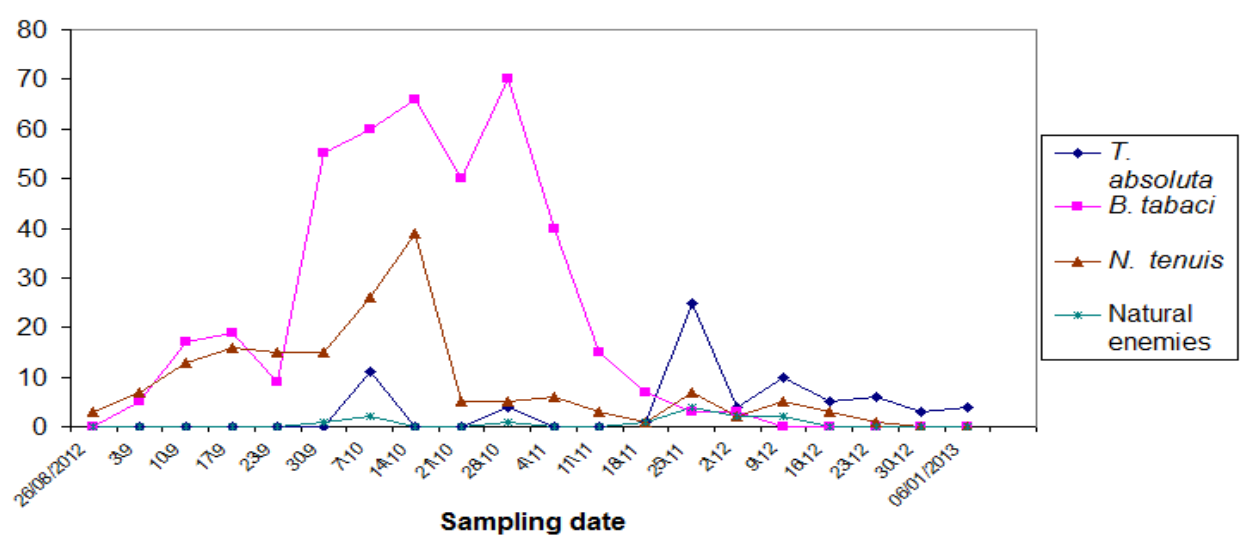

Fig (5): Weekly numbers of tomato pests and associated natural enemies during Nili plantations 2011 \& 2012

\section{Summer plantations 2012 and 2013:}

The population of predaceous insects was high especially in summer 2013. The total number of predators fluctuated during the period from April 28 and May 7 to July 1 and 23 with total number ranging between 1-6 indiv./10 leaves, the highest level of predaceous number was (6 indiv./ 10 leaves) during July 17 and 9 through summer 2012\2013, within the activity period of $T$. absoluta , B. tabaci and N. tenuis were found. as shown in Table 6 Fig 6.

Fayoum J. Agric. Res. \& Dev., Vol. 29, No.2, July, 2015 
Narmen .A. Youssef

Table (6): Weekly numbers of tomato pests and associated natural enemies during Summer plantations 2012 \& 2013

\begin{tabular}{|c|c|c|c|c|c|c|c|c|c|}
\hline \multicolumn{4}{|c|}{ Summer 2012 } & \multicolumn{5}{c|}{ Summer 2013 } \\
\hline $\begin{array}{c}\text { Sampling } \\
\text { date }\end{array}$ & $\begin{array}{c}\boldsymbol{T} \text {. } \\
\text { absoluta }\end{array}$ & $\begin{array}{c}\boldsymbol{B} . \\
\text { tabaci }\end{array}$ & $\begin{array}{c}\text { N. } \\
\text { tenuis }\end{array}$ & $\begin{array}{c}\text { Natural } \\
\text { enemies }\end{array}$ & $\begin{array}{c}\text { Sampling } \\
\text { date }\end{array}$ & $\begin{array}{c}\boldsymbol{T} . \\
\text { absoluta }\end{array}$ & $\begin{array}{c}\text { B. } \\
\text { tabaci }\end{array}$ & $\begin{array}{c}\text { N. } \\
\text { tenuis }\end{array}$ & $\begin{array}{c}\text { Natural } \\
\text { enemies }\end{array}$ \\
\hline $21 / 4 / 2012$ & 0 & 7 & 4 & 0 & $29 / 4 / 2013$ & 1 & 0 & 0 & 0 \\
\hline $28 / 4$ & 10 & 13 & 19 & 2 & $7 / 5$ & 0 & 0 & 15 & 1 \\
\hline $4 / 5$ & 5 & 10 & 29 & 2 & $16 / 5$ & 0 & 12 & 23 & 2 \\
\hline $11 / 5$ & 4 & 29 & 33 & 2 & $22 / 5$ & 3 & 29 & 28 & 5 \\
\hline $18 / 5$ & 14 & 50 & 37 & 2 & $29 / 5$ & 3 & 21 & 27 & 4 \\
\hline $26 / 5$ & 7 & 70 & 14 & 2 & $5 / 6$ & 2 & 30 & 4 & 5 \\
\hline $3 / 6$ & 6 & 77 & 0 & 0 & $12 / 6$ & 0 & 44 & 7 & 1 \\
\hline $10 / 6$ & 20 & 81 & 4 & 5 & $19 / 6$ & 7 & 40 & 13 & 2 \\
\hline $17 / 6$ & 14 & 23 & 50 & 6 & $26 / 6$ & 5 & 49 & 5 & 5 \\
\hline $24 / 6$ & 7 & 8 & 20 & 1 & $3 / 7$ & 9 & 62 & 39 & 5 \\
\hline $1 / 7$ & 5 & 0 & 27 & 1 & $9 / 7$ & 0 & 64 & 24 & 6 \\
\hline $9 / 7$ & 4 & 0 & 28 & 0 & $16 / 7$ & 0 & 40 & 4 & 3 \\
\hline $16 / 7$ & 0 & 0 & 13 & 0 & $23 / 7$ & 0 & 18 & 1 & 1 \\
\hline Total & 96 & 368 & 278 & 23 & Total & 30 & 409 & 190 & 40 \\
\hline Mean & 7.38 & 28.31 & 21.38 & 1.76 & Mean & 2.3 & 31.46 & 14.62 & 3.07 \\
\hline
\end{tabular}

Summer 2012
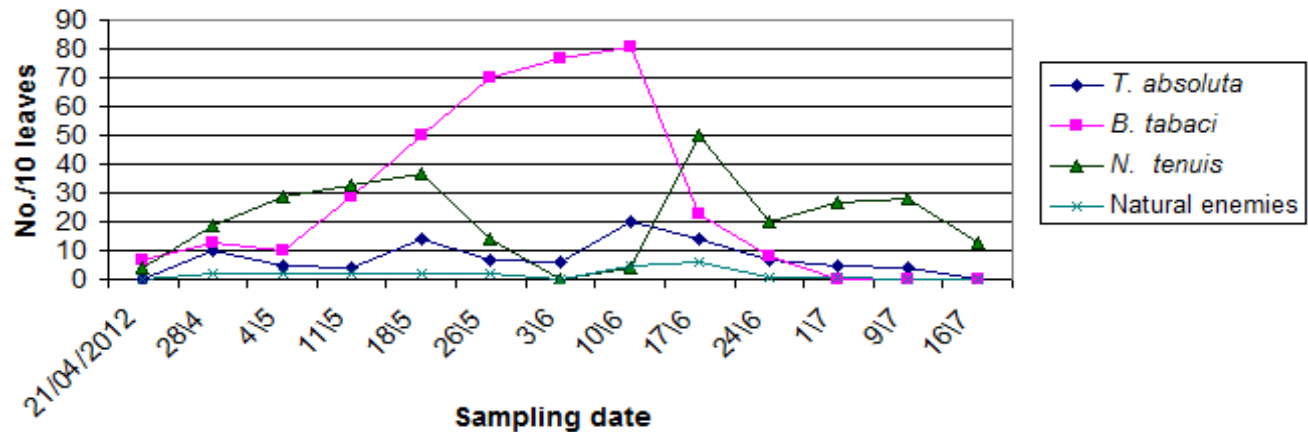

Summer 2013

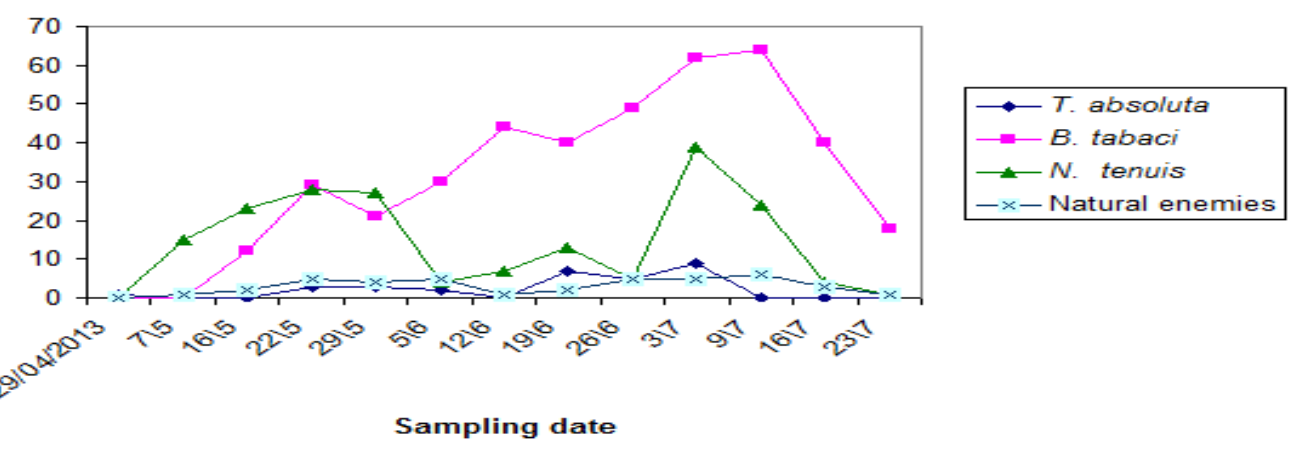

Fig (6): Weekly numbers of tomato pests and associated natural enemies during Summer plantations 2013.

Fayoum J. Agric. Res. \& Dev., Vol. 29, No.2, July, 2015 


\section{REFERENCES}

Abd-Ellah, S.H.A. (1994). Ecological studies on some important tomato insect pests and the susceptibility of different varieties of tomato to pests infestation. M. Sc. Thesis, Fac.Of Agric., Ain Shams Univ.

AL-Azawi, B.M. and AL-Azawi, A.F.(1988). Some observations on the tomato bug Engytatus tenuis Reut, (Miride :Hemiptera) in Baghdad Iraq J. Agric. Sci., ZANCO, 6(3): 85-88.

Andrew, G.S. Cuthbertson; James J. Mathers; Lisa F. Blackburn; Anastasia Korycinska; Weigiluo, Robert; J.Jacobson and Phil Northing (2013). Population development of Tuta absoluta (Meyrick) (Lepidoptera :Gelechiidae) under simulated UK glasshouse conditions. Insects, 4: 185- 197.

Barrientas, Z.R., Apablaza H.J., Norero, S.A. andEstay, P.P. (1988).Temperature base y constant termica de desarrollo de la polilla del tomate Tuta absoluta (Lepidoptera :Gelechiidae) Ciencia e Investigation Agraria 25: 133-137.

Chaudhuri,N., D.C. Deb and Senapati, S.K. (2001). Biology and fluctuation of whitefly (Bemisia tabaci Genn.) population on tomato as influenced by a biotic factors under terai region of west Bengal. Indian J. Agric. Res.,35(3): 155-160.

Cheerapha, P.( 2005).Thesis on effectiveness of local entomopathogenic fungi as bioinesticide for tomato insect pests.M.Sc. Thesis, Kasetsart University.

EPPO, (2005).Data sheet Tuta absoluta. OEPP/EPPO Bulletin 35: 434-435.

Fargalla, F.H. H. (2005). New approach for controlling some pests which infesting cucurbitaceae. M.Sc. Thesis, Fac. Of Sci., Mansoura Univ., $155 \mathrm{pp}$.

Germain, J.F., Lacordaire,A.I.,Concquempot, C., Ramel, J.M. andOudard E. (2009). Un nouveau ravageur de la tomate en France: Tuta absoluta . PHM- Revue Horticole 512: 37-41.

Lazgeen, H.A., Feyroz, R.H., Halgurd, R. I. and Salah, A. S. 2013. Population density of tomato leafminer Tutaabsoluta (Meyrick) (Lepidoptera: Gelechidae) under plastic houses conditions. Journal of Agriculture and veterinary science, s, 7-10.

Lopez, E. (1991) .Pollia del tomate: Proplema critic para la rentabilidaddelcultivo de verano. Empresa y Avance Agricola 1: 6-7.

Fayoum J. Agric. Res. \& Dev., Vol. 29, No.2, July, 2015 
Nannini, M., Alzori, F., Foddi, F., Pisci, R. and Sanna, F. 2011. A survey of Tuta absoluta (Meyrick)(Lepidoptera:Gelechidae) outbreaks in tomato green houses in southern Sardinia (Italy). XXVIII. International Horticultural Congress on Science and Horticulture for People (IHC 2010): International Symposium on Plant Protection.

Shaheen, A.H.(1983). Some ecological aspects of the whitefly, Bemisia tabaci (Genn.) on tomato. Bull. Soc. Ent. Egypt, 62: 83-87.

Shalaby, F.F., Abdel - Gawaad, A. A., EL-Sayed, A.M. and Abo - ELGhar, M.R.(1990). Natural role of Eretmocerus mundus Mercet and Prospaltella lutea Masi on populations of Bemisia tabaciGenn. Agric. Res. Rev. Cairo, 68(1): 197-208.

Shanab, L.M. and Awad - Allah, S.S. (1982). Studies on the whitefly, Bemisia tabaci (Genn.).infesting tomato at Mansoura district, Egypt. ActaPhytopathogica Academic Sclentiarum Nugaricae , $17(112)=147-155$.

Snedecor, W. and Cochran, W. (1990).Statistical methods. Iowa State University Press, AmerIowa, USA

Tantawy, Maha , A. (1995). Monitoring of Bemisia tabaci (Genn.) population on certain crops and its control. M. Sc. Thesis, of Agric. Cairo Unv.

دراسات إيكولوجية لثلاثة افات حشرية تهاجم نباتات الطماطم فى محافظة الفيوم

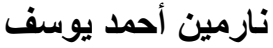

قسم وقاية النبات ـ كلية الزراعة - جامعة الفيوم

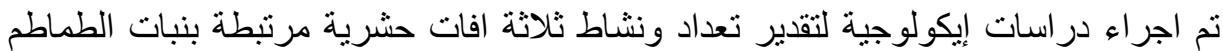

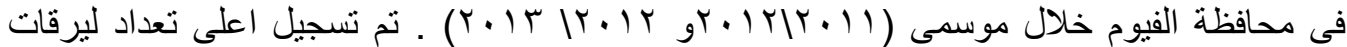

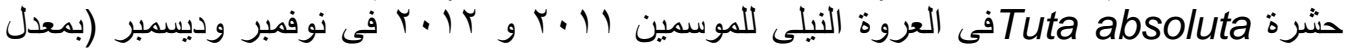

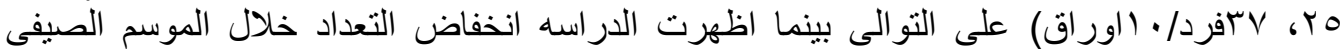

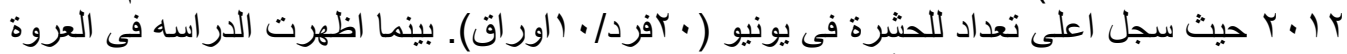

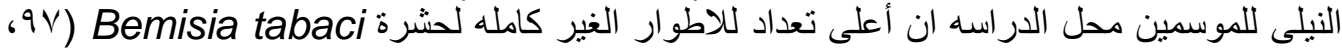

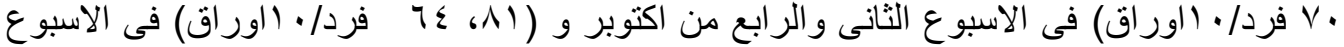

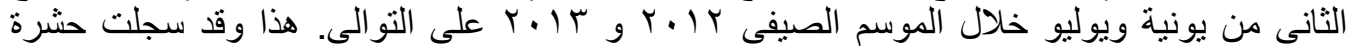
Nesidocoris tenuis

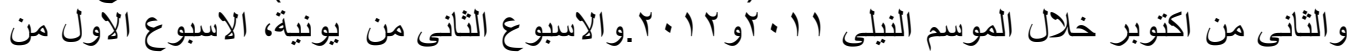

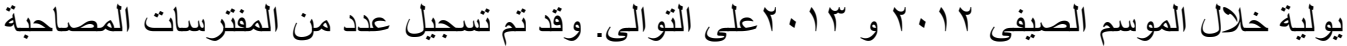

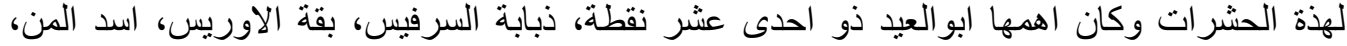

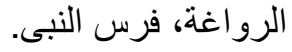

Fayoum J. Agric. Res. \& Dev., Vol. 29, No.2, July, 2015 